Acta Crystallographica Section C

Crystal Structure

Communications

ISSN 0108-2701

\section{A novel Janus-type AT nucleoside with benzoyl protecting groups forming a pleated-sheet structure}

\author{
Mei-Ying Pan, ${ }^{a}$ Xiao-Hua Wu, ${ }^{a}$ Dai-Bing Luo, ${ }^{b}$ Wen \\ Huang $^{\mathrm{a} *}$ and Yang $\mathrm{He}^{\mathrm{a} *}$
}

a Laboratory of Ethnopharmacology, Institute for Nanobiomedical Technology and Membrane Biology, Regenerative Medicine Research Center, West China Hospital, West China Medical School, Sichuan University, No. 1 Keyuansilu, Gaopeng Dadao, Chengdu, Sichuan 610041, People's Republic of China, and ' ${ }^{\mathbf{b}}$ Analytical and Testing Center, Sichuan University, No. 24 South Section 1, Yihuan Road, Chengdu 610064, People's Republic of China

Correspondence e-mail: huangwen@scu.edu.cn, heyangqx@yahoo.com.cn

Received 28 January 2011

Accepted 27 March 2011

Online 14 April 2011

The title compound, 5-amino-8-(2,3,5-tri- $O$-benzoyl- $\beta$-Dribofuranosyl)pyrimido[4,5- $d$ ]pyrimidine-2,4(3H,8H)-dione methanol monosolvate, $\mathrm{C}_{32} \mathrm{H}_{25} \mathrm{~N}_{5} \mathrm{O}_{9} \cdot \mathrm{CH}_{4} \mathrm{O}$, which crystallized slowly from methanol, exhibits an anti conformation with a glycosyl-bond torsion angle of $\chi=-141.28(17)^{\circ}$. The furanose moiety adopts an $N$-type sugar puckering $\left({ }^{3} T_{4}\right)$. The corresponding pseudorotation phase angle and maximum amplitude are $P=24.5(2)^{\circ}$ and $\tau_{\mathrm{m}}=38.3(2)^{\circ}$, respectively. In the solid state, one methanol molecule acts as a bridge joining adjacent nucleoside molecules head-to-head, leading to a pleated-ribbon supramolecular structure, with the base moieties located in the centre of the ribbon and the sugar residues protruding to the outside of the layers, as in a DNA helix. The pleated-ribbon supramolecular structure is tethered together into a two-dimensional infinite pleated-sheet structure through aromatic stacking between the nucleobase planes and the benzene rings of the benzoyl protecting groups on the $5^{\prime}-\mathrm{OH}$ group of furanose.

\section{Comment}

Recently, a novel Janus-type GC (J-GC) nucleoside, (II) (from the two-faced Roman god Janus), has been synthesized in our laboratory which showed antiviral potential (Yang et al., 2011). The base moiety of J-GC has one face with a Watson-Crick donor-donor-acceptor $(D D A)$ hydrogen-bond array of guanine and the other with an acceptor-acceptor-donor $(A A D)$ hydrogen-bond array of cytosine. In principle, J-GC could pair with cytidine or guanosine via rotation of the glycosyl bond. At the same time, this J-GC nucleoside can also associate through self-complementary hydrogen-bond formation. This property has been employed to construct nanoarchitectures such as trimers (Sessler et al., 2003), rosettes
(Marsh et al., 1996; Fenniri et al., 2001) and regular noncovalent polymer arrays (Asadi et al., 2007; Marsh et al., 1994). In order to make full use of these properties to generate different supramolecular structures in the field of nucleosides and oligonucleotides, we wish to expand these Janus nucleosides from a tridentate GC series to a bidentate AT series. Very few structures with the Janus-type AT base moiety have been reported (Tominaga et al., 1991; Asadi et al., 2007). Consequently, we synthesized the Janus-type AT nucleoside (J-AT); the full synthetic route will be reported elsewhere. The base moiety of J-AT has one face with a Watson-Crick acceptordonor $(A D)$ hydrogen-bond array of adenine and the other with a donor-acceptor $(D A)$ hydrogen-bond array of thymine. Interestingly, we have found for the first time that the solidstate structure of the benzoylated J-AT nucleoside, (I), can form a pleated-sheet suprastructure, a finding that we report in this paper. The expected hydrogen-bond patterns of (I) and (II) are shown in the scheme (arrows labelled $A$ represent hydrogen-bond acceptors and those labelled $D$ represent hydrogen-bond donors).

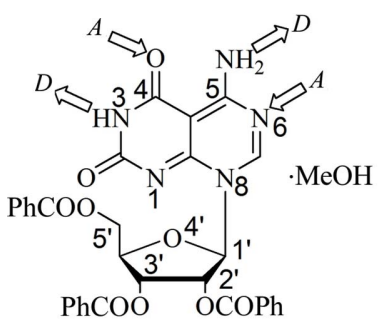

(I) J-AT

Systematic numbering

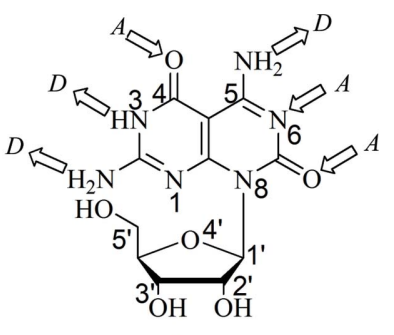

(II) $\mathrm{J}-\mathrm{GC}$
The structure of (I) is shown in Fig. 1, selected geometric parameters are listed in Table 1 and the hydrogen-bond geometry is listed in Table 2.

The pyrimido $[4,5-d]$ pyrimidine ring is almost planar. For the thymine pyrimidine ring, the deviations of the ring atoms from the $\mathrm{N} 1 / \mathrm{C} 2 / \mathrm{N} 3 / \mathrm{C} 4 / \mathrm{C} 10 / \mathrm{C} 9$ least-squares plane range from -0.009 (2) (for $\mathrm{C} 2$ ) to 0.016 (1) $\AA$ (for N1), with an r.m.s. deviation of $0.010 \AA$. The exocyclic atoms N11, O12 and O13 and the $\mathrm{C}^{\prime}$ substituent group deviate from this plane by -0.135 (4), 0.067 (3), -0.043 (3) and -0.189 (4) A, respectively. For the adenine pyrimidine ring, the deviations of the ring atoms from the $\mathrm{C} 10 / \mathrm{C} 9 / \mathrm{N} 8 / \mathrm{C} 7 / \mathrm{N} 6 / \mathrm{C} 5$ least-squares plane range from -0.017 (1) (for C5) to 0.012 (1) $\AA$ (for C10), with an r.m.s. deviation of $0.011 \AA$. The deviations of exocyclic atoms $\mathrm{N} 11, \mathrm{O} 12$ and $\mathrm{O} 13$ from this ring are $-0.135(4)$, 0.055 (4) and -0.129 (4) $\AA$, respectively. The dihedral angle between the two rings is $1.62(11)^{\circ}$. Therefore, the parameters of each ring of the pyrimido[4,5- $d]$ pyrimidine are quite close to those of canonical adenine (Lai \& Marsh, 1972) and thymine (Ozeki et al., 1969). The orientation of the nucleobase relative to the sugar ring (anti/syn conformation) in a normal N9-glycosylated purine nucleoside system is defined by the torsion angle $\chi\left(\mathrm{O}^{\prime}-\mathrm{Cl}^{\prime}-\mathrm{N} 9-\mathrm{C} 4\right)$ (IUPAC-IUB Joint Commission on Biochemical Nomenclature, 1983). When the pyrimidine ring of the purine is located outside the sugar plane the conformation is defined as anti, and when it is located 


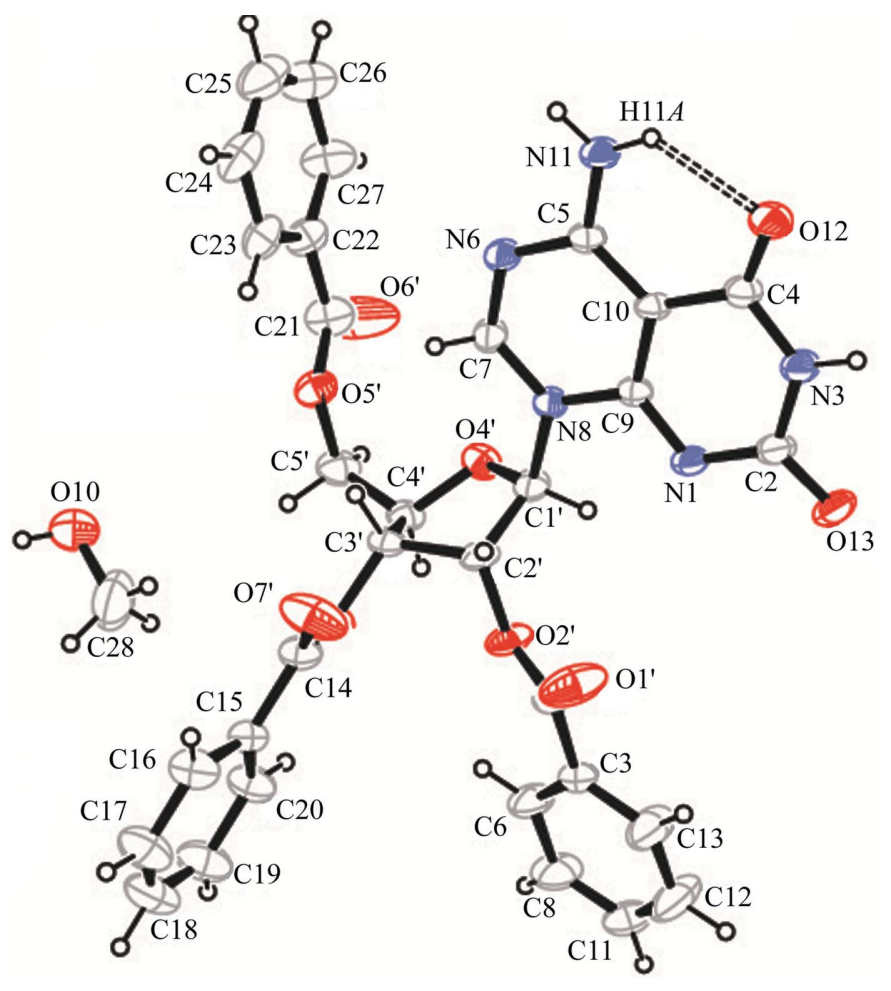

Figure 1

A perspective view of (I), showing the atom-labelling scheme and the intramolecular hydrogen bond (dashed line). Displacement ellipsoids are drawn at the $30 \%$ probability level.

inside the sugar plane the conformation is defined as syn. In the case of (I), the anti/syn conformation is also defined by the torsion angle $\chi\left(\mathrm{O}^{\prime}-\mathrm{C1}^{\prime}-\mathrm{N} 8-\mathrm{C} 9\right)$, which is $-141.28(17)^{\circ}$, with the thymine ring located outside the sugar ring. Therefore, (I) adopts an anti conformation. Another conformational parameter of interest is the puckering of the ribofuranose moiety, which is defined by the pseudorotation phase angle and the maximum puckering amplitude (Altona \& Sundaralingam, 1972). The values of these two parameters are $P=$ $24.5(2)^{\circ}$ and $\tau_{\mathrm{m}}=38.3(2)^{\circ}$, respectively. The corresponding sugar puckering mode is an asymmetrical twist of ${ }^{3} T_{4}$ in the north $\left(3^{\prime}\right.$-endo $)$ region, which is in the normal range for a pyrimidine or purine ribonucleoside system. The orientation of the exocyclic $5^{\prime}$-hydroxy group relative to the ribofuranose ring is characterized by the torsion angle $\gamma\left(\mathrm{O}^{\prime}-\mathrm{C}^{\prime}-\mathrm{C}^{\prime}-\right.$ $\left.\mathrm{C}^{\prime}\right)$, which is $57.3(3)^{\circ}$ for (I), corresponding to the $+s c$ (gauche) conformation.

A very interesting phenomenon is that the intermolecular connection of (I) is not mediated through self-complementary Watson-Crick base pairs as proposed for J-GC base pairs (Fenniri et al., 2001; Marsh et al., 1996; Yang et al., 2011) (see scheme). The unit cell of (I) consists of four nucleoside molecules and four methanol molecules (Fig. 2). Each nucleoside molecule is involved in six intermolecular hydrogen bonds and one intramolecular $\mathrm{N} 11-\mathrm{H} 11 A \cdots \mathrm{O} 12$ interaction (Table 2 and Fig. 3). Four intermolecular hydrogen bonds act as the linking units, connecting adjacent nucleosides head-to-head in an antiparallel manner to form an undulating ribbon in one

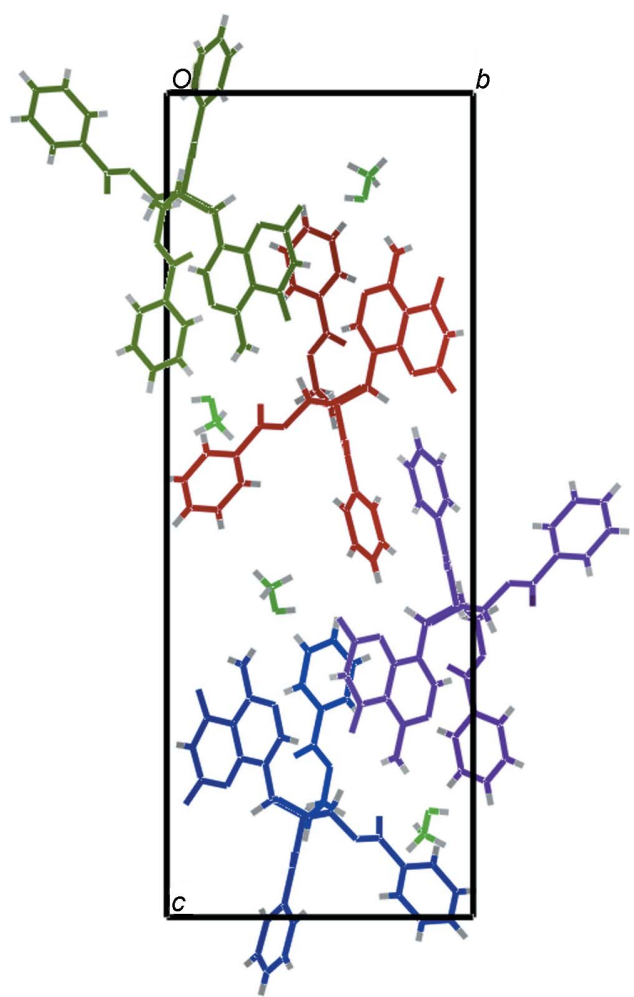

Figure 2

An arrangement of the nucleoside and methanol molecules in the unit cell of (I), viewed along [100]. Hydrogen bonds have been omitted for clarity.

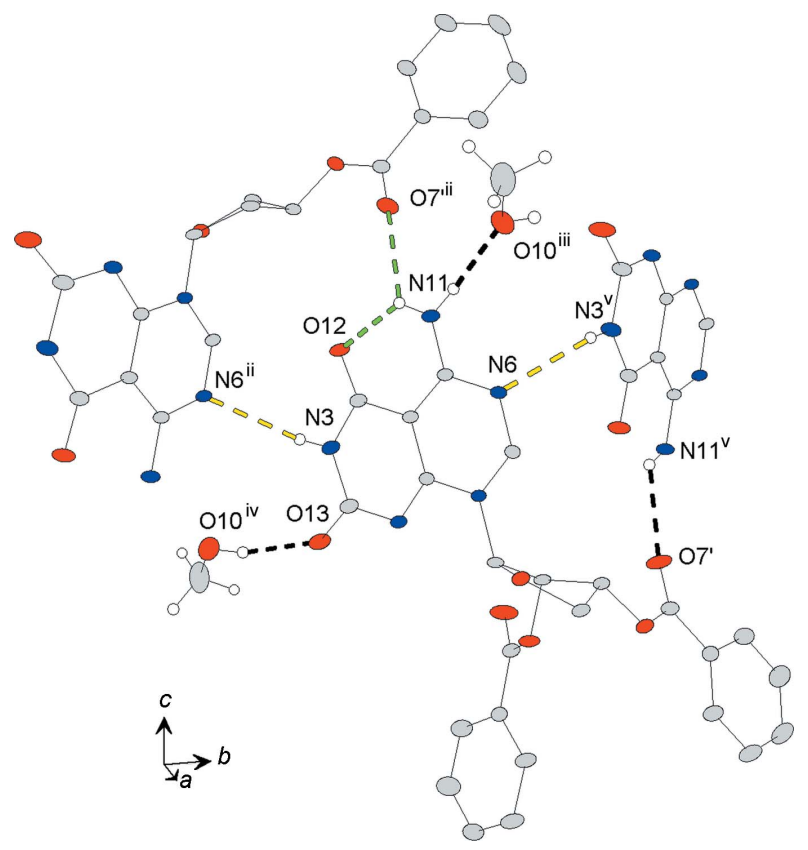

Figure 3

A detailed view of the hydrogen bonds surrounding one nucleoside molecule. Hydrogen bonds are indicated by dashed lines. (In the electronic version of the paper, the bifurcated hydrogen bonds are shown in green and the hydrogen bonds with large distances are shown in yellow; the remaining hydrogen bonds are shown in black.) $\mathrm{H}$ atoms of (I) and some groups which are not involved in hydrogen bonds have been omitted for clarity. (In the electronic version of the paper, atoms are coded as follows: red for oxygen, blue for nitrogen, grey for carbon and white for hydrogen.) [Symmetry codes: (ii) $-x, y-\frac{1}{2},-z+\frac{3}{2}$; (iii) $-x+1$, $y-\frac{1}{2},-z+\frac{3}{2}$; (iv) $-x, y+\frac{1}{2},-z+\frac{3}{2}$; (v) $x-1, y-1, z$.] 


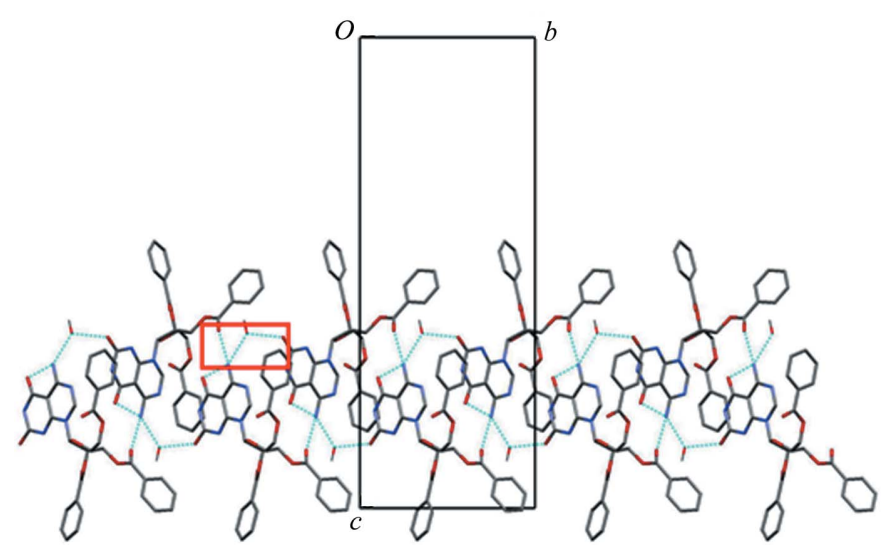

Figure 4

A packing diagram for (I), viewed along [100]. Hydrogen bonds are indicated by dashed lines. The hydrogen-bond motif in the small rectangular frame is the linking unit. $\mathrm{H}$ atoms have been omitted for clarity.

layer (Fig. 4). The N3-H3 - N6 hydrogen bond is not shown, owing to the large distance between atoms H3 and N6. Three of the linking units are highlighted by the small rectangle in Fig. 4: the first is formed between the $\mathrm{H}$ atom of the exocyclic amino group of one nucleoside molecule and the $\mathrm{O}$ atom of the benzoyl group of the previous nucleoside molecule in the chain; the second hydrogen bond is formed between the $\mathrm{H}$ atom of the exocyclic amino group of the nucleoside molecule and the $\mathrm{O}$ atom of the methanol molecule; and the third is formed between the $\mathrm{H}$ atom of the same methanol molecule and the exocyclic $\mathrm{O}$ atom of the next nucleoside molecule in the chain. This ribbon has a pleated linear structure, viewed along the [001] orientation, as shown in Fig. 5. This arrangement is quite different from the flattened structures reported for 5,7-diamino-1-heptylpyrimido[4,5- $d]$ pyrimidine-2,4(1H,3H)dione and 5,7-diamino-1-butyl-1 $H$-pyrido[4,3- $d$ ]pyrimidine2,4-dione (Asadi et al., 2007). In this pleated-ribbon structure, the base moieties are located in the centre of the ribbon and the sugar residues protrude to the outside of the layers (see

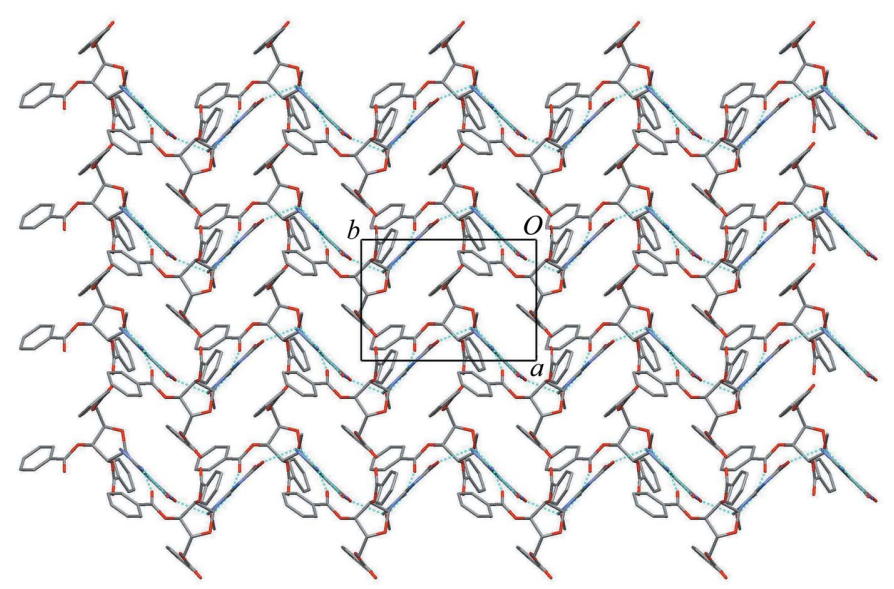

Figure 5

A four-layered packing diagram for (I), viewed along [001]. Hydrogen bonds are shown as dashed lines. $\mathrm{H}$ atoms have been omitted for clarity.
Fig. 4), as in a DNA helix. Finally, the layers are tethered together in a parallel fashion and stretched to form an infinite two-dimensional pleated-sheet structure through aromatic stacking between the nucleobase planes and the benzene rings of the benzoyl protecting groups on the $5^{\prime}-\mathrm{OH}$ group of furanose. Whether the special pleated-sheet structure exists at the RNA (or DNA) level will be explored in future studies.

\section{Experimental}

Compound (I) was synthesized from bis(methylsulfanyl)methylenecyanamide. The detailed synthetic procedure will be reported elsewhere. Compound (I) was dissolved in methanol at $323 \mathrm{~K}$, and cooled slowly in steps of $0.5 \mathrm{~K} \mathrm{~h}^{-1}$ to ambient temperature in an incubator, giving colourless needle-shaped crystals suitable for single-crystal X-ray diffraction. A selected crystal of compound (I) was protected in the mother liquor in a tiny glass tube and epoxy resin was used to minimize the loss of the solvent.

\section{Crystal data}

\section{$\mathrm{C}_{32} \mathrm{H}_{25} \mathrm{~N}_{5} \mathrm{O}_{9} \cdot \mathrm{CH}_{4} \mathrm{O}$ \\ $M_{r}=655.61$ \\ Orthorhombic, $P 2{ }_{1} 2_{1} 2_{1}$ \\ $a=8.2526(3) \AA$ \\ $b=11.9486(4) \AA$ \\ $c=32.1865(10) \AA$}

\section{Data collection}

Oxford Diffraction Xcalibur CCD diffractometer

Absorption correction: multi-scan [CrysAlis PRO (Oxford Diffraction, 2010); empirical spherical harmonics, imple-

\section{Refinement}

$R\left[F^{2}>2 \sigma\left(F^{2}\right)\right]=0.038$

$w R\left(F^{2}\right)=0.093$

$S=0.91$

6101 reflections

441 parameters

4 restraints absorption correction using

$$
\begin{aligned}
& V=3173.82(18) \AA^{3} \\
& Z=4 \\
& \text { Mo } K \alpha \text { radiation } \\
& \mu=0.10 \mathrm{~mm}^{-1} \\
& T=293 \mathrm{~K} \\
& 0.42 \times 0.36 \times 0.20 \mathrm{~mm}
\end{aligned}
$$

$$
\begin{aligned}
& \text { mented in the } S C A L E 3 \\
& A B S P A C K \text { scaling algorithm] } \\
& T_{\min }=0.443, T_{\max }=1.0 \\
& 9151 \text { measured reflections } \\
& 6101 \text { independent reflections } \\
& 4128 \text { reflections with } I>2 \sigma(I) \\
& R_{\text {int }}=0.019
\end{aligned}
$$
$\mathrm{H}$ atoms treated by a mixture of independent and constrained refinement
$\Delta \rho_{\max }=0.20{\mathrm{e} \AA^{-3}}^{-3}$
$\Delta \rho_{\min }=-0.17$ e $\AA^{-3}$

In the absence of suitable anomalous scattering, Friedel equivalents cannot be used to determine the absolute structure. The Flack parameter (Flack, 1983) has an inconclusive value of 0.3 (9) with 2423 Friedel pairs. The known configuration of the D-ribofuranose derivatives was used to define the enantiomer employed in the refined model. The amine $\mathrm{H}$ atoms at $\mathrm{N} 11$ were located in a difference Fourier map and were refined using $\mathrm{N}-\mathrm{H}$ bond-length restraints of $0.86(2) \AA$, a $\mathrm{H} \cdots \mathrm{H}$ distance restraint of $1.40(4) \AA$, similarity

Table 1

Selected torsion angles $\left(^{\circ}\right)$.

\begin{tabular}{lclc}
\hline $\mathrm{O}^{\prime}-\mathrm{C} 1^{\prime}-\mathrm{C} 2^{\prime}-\mathrm{C} 3^{\prime}$ & $-19.90(18)$ & $\mathrm{C} 4-\mathrm{N} 3-\mathrm{C} 2-\mathrm{O} 13$ & $179.1(2)$ \\
$\mathrm{O}^{\prime}-\mathrm{C} 4^{\prime}-\mathrm{C} 5^{\prime}-\mathrm{O} 5^{\prime}$ & $-60.7(2)$ & $\mathrm{C} 4-\mathrm{C} 10-\mathrm{C} 5-\mathrm{N} 6$ & $-179.49(17)$ \\
$\mathrm{O} 12-\mathrm{C} 4-\mathrm{C} 10-\mathrm{C} 9$ & $-177.1(2)$ & $\mathrm{C} 7-\mathrm{N} 6-\mathrm{C} 5-\mathrm{N} 11$ & $177.5(2)$ \\
$\mathrm{N} 1-\mathrm{C} 9-\mathrm{C} 10-\mathrm{C} 5$ & $178.17(19)$ & $\mathrm{C} 7-\mathrm{N} 8-\mathrm{C} 1^{\prime}-\mathrm{O} 4^{\prime}$ & $34.4(3)$ \\
$\mathrm{N} 8-\mathrm{C} 9-\mathrm{C} 10-\mathrm{C} 4$ & $-178.65(16)$ & $\mathrm{C} 9-\mathrm{N} 1-\mathrm{C} 2-\mathrm{O} 13$ & $-177.1(2)$ \\
$\mathrm{C} 2-\mathrm{N} 3-\mathrm{C} 4-\mathrm{O} 12$ & $177.2(2)$ & $\mathrm{C} 9-\mathrm{N} 8-\mathrm{C} 1^{\prime}-\mathrm{O} 4^{\prime}$ & $-141.28(17)$ \\
$\mathrm{C} 3^{\prime}-\mathrm{C} 4^{\prime}-\mathrm{C}^{\prime}-\mathrm{O}^{\prime}$ & $57.3(3)$ & $\mathrm{C} 9-\mathrm{C} 10-\mathrm{C} 5-\mathrm{N} 11$ & $-176.94(19)$ \\
\hline
\end{tabular}




\section{organic compounds}

Table 2

Hydrogen-bond geometry $\left(\AA,^{\circ}\right)$.

\begin{tabular}{lllll}
\hline$D-\mathrm{H} \cdots A$ & $D-\mathrm{H}$ & $\mathrm{H} \cdots A$ & $D \cdots A$ & $D-\mathrm{H} \cdots A$ \\
\hline $\mathrm{O} 10-\mathrm{H} 10 \cdots \mathrm{O} 13^{\mathrm{i}}$ & 0.82 & 1.86 & $2.661(3)$ & 166 \\
$\mathrm{~N} 3-\mathrm{H} 3 \cdots \mathrm{N}^{\mathrm{ii}}$ & 0.86 & 2.40 & $3.113(2)$ & 141 \\
$\mathrm{~N} 11-\mathrm{H} 11 B \cdots \mathrm{O} 10^{\mathrm{iii}}$ & $0.87(1)$ & $1.96(1)$ & $2.835(3)$ & $176(2)$ \\
$\mathrm{N} 11-\mathrm{H} 11 A \cdots \mathrm{O} 7^{\text {ii }}$ & $0.86(1)$ & $2.29(2)$ & $2.860(2)$ & $124(2)$ \\
$\mathrm{N} 11-\mathrm{H} 11 A \cdots \mathrm{O} 12$ & $0.86(1)$ & $2.12(2)$ & $2.751(2)$ & $130(2)$ \\
\hline
\end{tabular}

Symmetry codes: (i) $x+1, y+1, z$; (ii) $-x, y-\frac{1}{2},-z+\frac{3}{2}$; (iii) $-x+1, y-\frac{1}{2},-z+\frac{3}{2}$.

restraints (s.u. of $0.02 \AA$ ) for the two C5 $\cdots \mathrm{H}$ distances and fixed $U_{\text {iso }}(\mathrm{H})$ values of $0.05 \AA^{2}$. All other $\mathrm{H}$ atoms were placed in geometrically idealized positions and treated as riding. For the hydroxy and methyl groups, $\mathrm{O}-\mathrm{H}=0.82 \AA$ and $\mathrm{C}-\mathrm{H}=0.96 \AA$, with $U_{\text {iso }}(\mathrm{H})=1.5 U_{\text {eq }}(\mathrm{O}, \mathrm{C})$. For the remaining groups, $\mathrm{C}-\mathrm{H}=0.93$ (aromatic), 0.97 (methylene) or $0.98 \AA$ (methine) and $\mathrm{N}-\mathrm{H}=0.86 \AA$, with $U_{\text {iso }}(\mathrm{H})=1.2 U_{\text {eq }}(\mathrm{C}, \mathrm{N})$.

Data collection: CrysAlis PRO (Oxford Diffraction, 2010); cell refinement: CrysAlis PRO; data reduction: CrysAlis PRO; program(s) used to solve structure: SHELXS97 (Sheldrick, 2008); program(s) used to refine structure: SHELXL97 (Sheldrick, 2008); molecular graphics: $O L E X 2$ (Dolomanov et al., 2009); software used to prepare material for publication: $O L E X 2$.

This work was supported by the National Natural Science Foundation of China (grant No. 20772087). The authors are grateful to the Analytical and Testing Center of Sichuan
University for support of the X-ray laboratory and Mr ZhiHua Mao for kindly helping with the X-ray data analysis.

Supplementary data for this paper are available from the IUCr electronic archives (Reference: QS3001). Services for accessing these data are described at the back of the journal.

\section{References}

Altona, C. \& Sundaralingam, M. (1972). J. Am. Chem. Soc. 94, 8205-8212.

Asadi, A., Patrick, B. O. \& Perrin, D. M. (2007). J. Org. Chem. 72, 466-475.

Dolomanov, O. V., Bourhis, L. J., Gildea, R. J., Howard, J. A. K. \& Puschmann, H. (2009). J. Appl. Cryst. 42, 339-341.

Fenniri, H., Mathivanan, P., Vidale, K. L., Sherman, D. M., Hallenga, K., Wood, K. V. \& Stowell, J. G. (2001). J. Am. Chem. Soc. 123, 3854-3855.

Flack, H. D. (1983). Acta Cryst. A39, 876-881.

IUPAC-IUB Joint Commission on Biochemical Nomenclature (1983). Eur. J. Biochem. 131, 9-15.

Lai, T. F. \& Marsh, R. E. (1972). Acta Cryst. B28, 1982-1989.

Marsh, A., Nolen, E., Gardinier, K. \& Lehn, J. (1994). Tetrahedron Lett. 35, 397-400.

Marsh, A., Silvestri, M. \& Lehn, J.-M. (1996). Chem. Commun. pp. 15271528.

Oxford Diffraction (2010). CrysAlis PRO. Version 1.171.33.66. Oxford Diffraction Ltd, Yarnton, Oxfordshire, England.

Ozeki, K., Sakabe, N. \& Tanaka, J. (1969). Acta Cryst. B25, 1038-1045.

Sessler, J., Jayawickramarajah, J., Sathiosatham, M., Sherman, C. \& Brodbelt, J. (2003). Org. Lett. 5, 2627-2630.

Sheldrick, G. M. (2008). Acta Cryst. A64, 112-122.

Tominaga, Y., Ohno, S., Kohra, S., Fujito, H. \& Mazurae, H. (1991). J. Heterocycl. Chem. 28, 1039-1042.

Yang, H.-Z., Pan, M.-Y., Jiang, D.-W. \& He, Y. (2011). Org. Biomol. Chem. 9 $1516-1522$. 